Rev. Elev. Méd. vét. Pays trop., 1976, 29 (3) : 195-198.

\title{
Enquête épidémiologique sur l'anémie infectieuse des équidés au Sénégal
}

\author{
par C. LE JAN $\left({ }^{*}\right)$, B. TOMA $\left({ }^{* *}\right)$ et P. BOURDIN $\left({ }^{*}\right)$
}

\begin{abstract}
RÉSUMÉ
Une enquête épidémiologique portant sur 1232 sérums d'équidés prélevés en 1972 et 916 en 1975 dans diverses régions du Sénégal y a révélé l'existence de l'infection inapparente du cheval par le virus de l'anémie infectieuse des équidés. L'incidence en est très fajble, inférieure à $1 \mathrm{p}$. 100. Un dépistage sérologique chez certaines catégories de chevaux et le contrôle des animaux importés devraient permettre de maintenir l'infection à un taux très bas.
\end{abstract}

L'anémie infectieuse des équidés (A.I.E.), maladie virale touchant essentiellement les chevaux et à un moindre degré les ânes, a donné lieu au cours de ces dernières décennies à de nombreux travaux.

Son intérêt pour le vétérinaire et l'éleveur tient à certaines particularités. La maladie évolue souvent sous forme chronique, entrecoupée d'épisodes aigus (4). Après infection par le virus, l'animal peut rester porteur et contagieux toute sa vie. La transmission se fait essentiellement par insectes hématophages ou accidentellement par piqûres en série avec la même seringue.

La détection des animaux infectés est facile depuis la mise au point d'un test sérologique simple, spécifique et rapide (3). Dans certains pays (U.S.A., Canada, Italie...), les mesures de lutte s'appliquent à tous les animaux fournissant une réponse positive au test de Coggins, qu'ils soient cliniquement sains ou malades. En France, seule l'A.I.E. cliniquement exprimée est classée maladie légalement contagieuse. Un nombre croissant de pays (U.S.A., Canada,

(*) Service de Virologie, Laboratoire national de l'Elevage et de Recherches vétérinaires (I.S. R. A.), Dakar-Hann (Sénégal).

(**) Ecole nationale vétérinaire d'Alfort, 94701 Maisons-Alfort (France).
Italie, Chili, etc...) exigent à l'importation un certificat garantissant l'absence d'anticorps précipitants anti-A.I.E. chez les équidés.

En Afrique, la maladie a été signalée autrefois dans le Maghreb (1) (7), en Erythrée (2) et en République Sud-Africaine (5). Récemment l'analyse de sérums, provenant du Sénégal, au laboratoire des Maladies contagieuses de l'École d'Alfort, a révélé plusieurs réactions positives alors que la maladie n'avait jamais été identifiée auparavant au Sénégal. Dans ce pays, le commerce du cheval connaît une activité non négligeable, en raison de l'existence, d'une part, d'un effectif assez important de chevaux locaux utilisés pour la traction animale et, d'autre part, d'un dépôt d'étalons à Dara qui a permis le développement de croisements et la production de sujets de valeur pour le sport hippique. Par suite, il nous a paru intéressant de faire une enquête sur la fréquence de l'anémie infectieuse au Sénégal. Ce sont les résultats de cette étude que nous rapportons dans les lignes qui suivent.

\section{MATÉRIEL ET METHODES}

\section{- Test d'immunodiffusion en gélose}

Les anticorps sériques spécifiques de l'A.I.E. sont mis en évidence par la technique classique 
d'immunodiffusion en gélose (6). L'antigène de référence et le sérum positif sont préparés au laboratoire des maladies contagieuses de l'École nationale vétérinaire d'Alfort. Une partie des sérums a été étudiée à Alfort et l'autre à Dakar.

\section{- Sérums éprouvés}

L'étude a porté sur un lot de sérums prélevés en 1971-1972 et conservés à $-20^{\circ} \mathrm{C}$ et sur des prélèvements effectués en 1975.

Au Sénégal, la limite inférieure de l'élevage du cheval est marquée par l'isohyète mille, correspondant au début des régions infectées de trypanosomiase (fig. 1). Dans l'ensemble du pays, on trouve des chevaux utilisés pour la traction agricole et, dans les agglomérations, des chevaux de charrette. Ces chevaux sont, en général, des mâles importés du Mali, la production du cheval de race commune étant très réduite. Les sujets importés du Mali sont regroupés et distribués à partir des marchés de Kaolack.

Autour de Dara, en région sahélienne où se trouve le principal haras national qui possède plusieurs pur-sang (Arabes, Anglais, Angloarabes), est située la zone de production des chevaux de selle. Des sujets de valeur, de course, de sport et de prestige, sortent de ces élevages.

Un effectif assez important de chevaux de selle, originaires de Dara est regroupé dans la région du Cap Vert : 150 chevaux répartis en trois clubs hippiques, 200 chevaux à la garde nationale et une centaine dans les écuries de course.

La figure 1 indique la répartition géographique des prélèvements qui ont couvert les différents types de chevaux existant au Sénégal.

\section{RÉSULTATS}

\section{- Prélèvements de 1972}

1232 sérums ont été examinés. 11 sont positifs. Le taux d'infection est de 0,9 p. 100.

- Prélèvements de 1975

Sur 916 sérums, 5 ont réagi positivement (tabl. $n^{\circ}$ I). Le taux d'infection est de 0,5 p. 100.

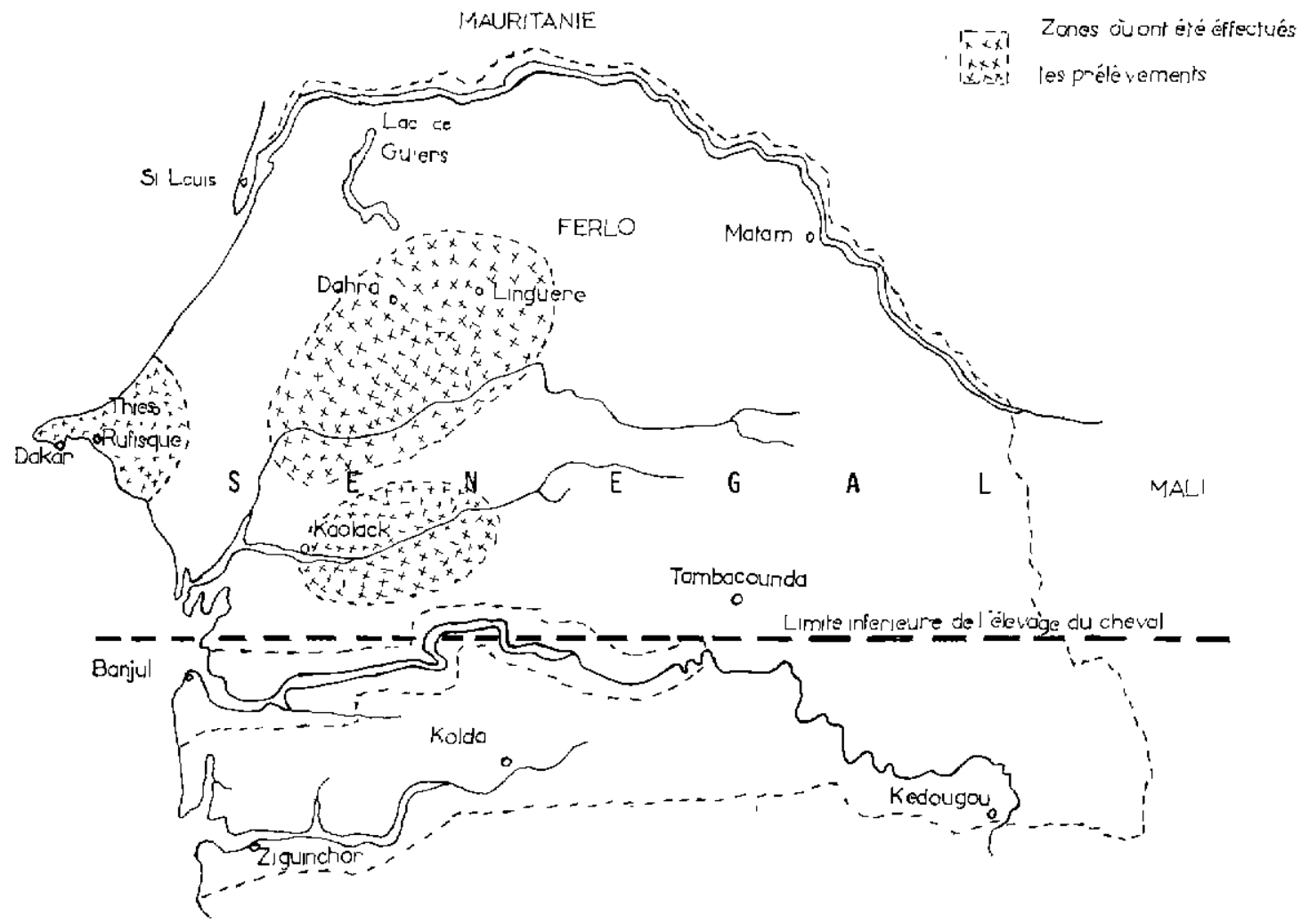

Représentation schématique du Sénégal montrant les zones où ont été effectués les prélèvements de sérum d'équidés. 
TABL. $N^{\circ} I-k e ́ s u l t a t s$ du test de Coggins effectuê sur les sérums prélevés en 1975.

\begin{tabular}{|l|c|c|}
\hline $\begin{array}{c}\text { Origine géographique } \\
\text { des prêlềvements }\end{array}$ & $\begin{array}{c}\text { Nombre de } \\
\text { sérums } \\
\text { êprouvés }\end{array}$ & $\begin{array}{c}\text { Nombre de } \\
\text { gérums } \\
\text { positifs }\end{array}$ \\
\hline Cap Vert & 465 & 2 \\
\hline Sine-Saloum & 223 & 2 \\
\hline Région de Diourbel & 186 & 1 \\
\hline Région du Fleuve & 42 & 0 \\
\hline Total & 916 & 5 \\
\hline
\end{tabular}

\section{DISCUSSION}

La preuve de l'existence de l'A. I. E. au Sénégal est donc apportée ; cependant l'incidence est très faible (inférieure à 1 p. 100).

Les conditions optimales de transmission de l'A. I. E. sont réunies au Sénégal, à savoir la pullulation saisonnière d'insectes hématophages, et pourtant le taux d'infection reste très faible. Il est à noter qu'un cheval, réagissant positivement, vit depuis 8 ans en compagnie d'un autre qu'il n'a pas contaminé.

Aucun cas clinique n'a encore été identifié, bien que les sérums d'animaux malades qui nous parviennent et pour lesquels on pourrait soupçonner l'A. l. E. soient soumis à la réaction de Coggins.

Une situation épidémiologique semblable existe dans différents pays d'Afrique, car l'un d'entre nous a obtenu, au cours de ces dernières années, des réponses sérologiques positives sur des chevaux vivant dans des pays comme le Maroc, l'Éthiopie et le Tchad, où la maladie n'est pas reconnue cliniquement.

Des résultats de cette enquête, nous pouvons tirer des données pratiques.

L'A. I. E. étant présente au Sénégal, mais de façon rare et inapparente, il serait, en théorie, facile de l'éliminer totalement par l'abattage des quelques animaux réagissant au test de Coggins et par un contrôle strict aux frontières. Ceci n'est cependant pas réalisable actuellement. Aux difficultés de surveillance des importations de chevaux, s'ajouteraient les réticences des éleveurs qui refuseraient de laisser sacrifier des animaux en bonne santé apparente.

La production du cheval de sport est localisée ; une recherche d'anticorps anti-A. I. E. sur les juments avant chaque insémination à partir du sperme d'étalons des haras nationaux et sur les produits lors de leur homologation, ainsi que sur les chevaux de selle importés, permettrait de maîtriser l'évolution de l'A. I. E. et de circonscrire rapidement tout foyer sans mettre en cuvre des moyens trop lourds pour une infection dont l'incidence économique est sans doute faible.

\section{CONCLUSION}

Cette enquête confirme le caractère ubiquitaire de l'anémie infectieuse des équidés. Le taux d'infection au Sénégal est très faible

TABL. N*II-Examen dêtaillé et comparatif des résultatr obtenus sur les sếrums prélevês en 1975

\begin{tabular}{|c|c|c|}
\hline $\begin{array}{c}\text { Lieu de } \\
\text { prélëvement. }\end{array}$ & Types de chevaux éprouvês & observation \\
\hline Cap Vert & $\begin{array}{l}\text { - Chevaux de la Gendarmerie } \\
\text { - Cercles hippiques } \\
\text { (150 chevaux) }\end{array}$ & $\begin{array}{l}\text { 1975: } 2 \text { sérums positifs : } \\
1 \text { cheval de charrette à Thiès } \\
1 \text { cheval de charrette à Rufisque; ce cheval, } \\
\text { hors d'âge, vit depuis } 8 \text { ans avec un autre } \\
\text { qu'il n'a pas contaminé. }\end{array}$ \\
\hline Sine-Saloum & $\begin{array}{l}\text { - Dêpôt d'étalona de pur-sang } \\
\text { de Kaolack } \\
\text { - Chevaux de charrette } \\
\text { - Chevaux arrivant du Mali. }\end{array}$ & $\begin{array}{l}1975 \text { : } 2 \text { sérums pooitifs : } \\
1 \text { cheval de charrette âgê de } 6 \text { ans à Guirguênoé } \\
1 \text { cheval âgé de } 6 \text { ans, arrivant du Mali. }\end{array}$ \\
\hline $\begin{array}{l}\text { Région de } \\
\text { Diourbe } 1\end{array}$ & $\begin{array}{l}\text { - Dépât d'étalons de Dara et } \\
\text { élevages de chevaux de course } \\
\text { et de sport environnanta. } \\
\text { - Chevaux de Linguère, Louga, } \\
\text { Bambey (chevaux de craction) }\end{array}$ & $\begin{array}{l}1975 \text { : Le seul cheval ayant réagi positivement est une } \\
\text { jument de } 7 \text { ans, dens la rếgion de Dara. Certe } \\
\text { jument appartient une zone d'êlevage de chevaux } \\
\text { de course, obtenus par croísements de chevaux } \\
\text { d'origine locale avec des étalons de sang. }\end{array}$ \\
\hline
\end{tabular}

Rectificatif : Colonne « Observations », 70 ligne, lire Guınguinéo. 
(inférieur à 1 p. 100) et l'infection ne semble pas s'être exprimée cliniquement jusqu'à présent. Les insectes hématophages étant saisonnièrement très fréquents au Sénégal, il est surprenant que le niveau d'infection soit si faible. Dans d'autres pays à climat tropical, en particulier en Amérique Centrale et en Amérique du Sud, l'incidence de la maladie est beaucoup plus élevée.

L'anémie infectieuse des équidés ne pose pas pour l'instant un réel problème pathologique au Sénégal. Il n'en reste pas moins qu'elle y existe et pourrait s'y développer lorsque les élevages de chevaux auront atteint une plus grande concentration. Mais, surtout, les résultats de cette enquête placent le Sénégal sur la liste des pays où l'A. I. E. a été reconnue. et le conduisent donc à s'aligner sur les mesures prises à l'étranger, en vue d'échanges commerciaux.

\section{SUMMARY}

Epidemiological survey on equine infectious anaemia in Senegal.

An epidemiological study, on 1232 horse serums samples collected in 1972 and 916 collected in 1975 in several countries of Senegal, revealed occult infection of horses by equine infectious anaemia virus. Its incidence is very low (under 1 p. 100). Systematical serologic examinations on imported horses and some local equine classes would allow to maintain infection to a very low level.

\section{RESUMEN}

\section{Encuesta epidemiologica sobre la anemia infecciosa equìna en Senegal}

Una encuesta epidemiologica efectuada con 1232 sueros de equinos recogidos en 1972 y 916 en 1975 en varias regiones de Senegal mostró la existencia de la infección inaparente del caballo por el virus de la anemia infecciosa equina.

La incidencia es poco elevada, inferior a 1 p. 100.

Un despiste serologico en ciertas categorias de caballos y la comprobación de los animales importados deberian de permitir el mantenimiento de una tasa de infección muy baja.

\section{BIBLIOGRAPHIE}

1. BALOZET (L.) et AOUSTIN (L.). Premier cas d'anémie unfectieuse des équidés, observé en Tunisie. Arch. Inst. Pasteur Tunis, 1934, $23: 360$.

2. CILLI (V.). Studio de un focolaio di anemia infettiva dell'asino in Eritrea. Nuova Vet., 1938, $16: 179$.

3. COGGINS (L.) et NORCROSS (N. L.). Immunodiffusion reaction in equine infectious anemia. Cornell Vet., $1970,60: 330$.

4. GORET (P.), MICHEL (C.) et TOMA (B.). L'anémie infectieuse des équidés. Paris, l'Expansion, 1968. (Les maladies animales à virus.)
5. THEILER (A.) et KEHOE (D.). Infectious or pernicious anemia of equines in South Africa. 3rd and 4th Report, Director Vet. Research, Dept. Agric. South Africa.-Pretoria, 1915, p. 217.

6. TOMA (B.), LUKA-ISKANDER (G. E.) et GORET (P.). Sérodiagnostic de l'anémıe infectieuse des équidés par précipitation en gélose. I. Mise au point de la technique. Bull. Acad. vét., 1971, 44: 403.

7. VELU (H.). Les anémies pernicieuses du cheval au Maroc et les diagnostics biologiques. Bull. Soc. cent. Méd. vét., 1921, 74: 263. 